\title{
Discrepancy between modelled and measured radial electric fields in the scrape-off layer of divertor tokamaks: a challenge for $2 \mathrm{D}$ fluid codes ?
}

\author{
A.V. Chankin ${ }^{1}$, D.P.Coster ${ }^{1}$, N.Asakura ${ }^{2}$, X.Bonnin ${ }^{3}$, G.D.Conway ${ }^{1}$, G.Corrigan ${ }^{4}$, \\ S.K.Erents ${ }^{4}$, W.Fundamenski ${ }^{4}$, J.Horacek ${ }^{5}$, A.Kallenbach ${ }^{1}$, M.Kaufmann ${ }^{1}$, C.Konz ${ }^{1}$, \\ K.Lackner ${ }^{1}$, H. W. Müller ${ }^{1}$, J.Neuhauser ${ }^{1}$, R.A.Pitts ${ }^{6}$, M.Wischmeier ${ }^{1}$ \\ ${ }^{1}$ Max-Planck-Institut für Plasmaphysik, EURATOM Association, D-85748 Garching, \\ Germany \\ 2 Japan Atomic Energy Agency (JAEA); Naka Fusion Research Institute, 801-1 Mukouyama, \\ Naka-Machi, Ibaraki-ken, 311-0193, Japan \\ ${ }^{3}$ CNRS-LIMHP, Université Paris 1399 AvenueJ.-B. Clément, F-93430 Villetaneuse, France \\ ${ }^{4}$ EURATOM-UKAEA Fusion Association, Culham Science Centre, Abingdon, Oxfordshire, \\ OX14 3DB, UK \\ ${ }^{5}$ Institute of Plasma Physics, Za Slovankou 3, 182 00, Prague 8, Czech Republic \\ ${ }^{6}$ Centre de Recherches en Physique des plasmas, Association EURATOM-Confédération \\ Suisse, École Polytechnique Fédérale de Lausanne, Ch-1015, Switzerland
}

\begin{abstract}
Examination of radial electric field $\left(\mathrm{E}_{\mathrm{r}}\right)$ profiles in the scrape-off layer (SOL) of ASDEX Upgrade (AUG) and JET revealed large discrepancies between 2D fluid edge modelling and experiment. Experimental profiles of plasma potential $\left(\mathrm{V}_{\mathrm{p}}\right)$ in the outer (low field) side of the plasma, obtained with reciprocating Langmuir probes, decay radially with electron temperature, $\mathrm{T}_{\mathrm{e}}$, with the $-\mathrm{eE}_{\mathrm{r}} / \nabla \mathrm{T}_{\mathrm{e}}$ ratio being $>1.5$. In contrast, code simulated $\mathrm{E}_{\mathrm{r}}$ are fairly low in most of the SOL (compared to $-\nabla \mathrm{T}_{\mathrm{e}} / \mathrm{e}$ ). Modelling with kinetic treatment of neutrals and drifts was performed using the SOLPS code for AUG cases and EDGE2D-Nimbus for JET cases.
\end{abstract}

Mismatches between modelled and experimental $E_{\mathrm{r}}$ may be caused by the recently established tendency for the SOLPS code to underestimate $T_{e}$ in the divertor of AUG. It was attributed to non-locality of parallel transport of supra-thermal, heat-carrying electrons originating upstream of the divertor, which are usually only weakly collisional and can penetrate, with few collisions, to the target. Ratios $-\mathrm{eE}_{\mathrm{r}} / \nabla \mathrm{T}_{\mathrm{e}}$ obtained from the probe measurements in JET are of order 1.6, while in AUG, JT-60U and TCV they are of order 3. Such high values point to the possibility of fast electrons contributing, apart from target heat fluxes, also to the formation of the Debye sheath.

The problem of the underestimation of $E_{r}$ in the codes must be closely related with the wellknown problem of the underestimation of those parts of parallel ion flows in the SOL that are influenced by the toroidal field direction. It was demonstrated earlier that parallel ion flow at the outer midplane is dominated by the ion Pfirsch-Schlüter flow, which in turn is partly driven by the radial electric field. The $T_{e}$ and $E_{r}$ discrepancies, as well as discrepancies between simulated and experimental parallel ion flows, put into question the validity of fluid codes for the plasma edge modelling and prompt the inclusion of kinetic effects into presentday 2D fluid codes which assume strong collisionality. 


\section{Introduction}

Recent detailed comparison between ASDEX Upgrade (AUG) experimental data and results of the SOLPS code simulations revealed the tendency for the code solutions to underestimate the target electron temperature and overestimate its density [1,2]. Sensitivity studies of the SOLPS solutions to various assumptions in both plasma and neutral models have almost entirely eliminated the possibility of inappropriate description of neutrals or ions as the cause of discrepancies between the code and experiment [1-3]. The discrepancies are likely to be attributed to supra-thermal electrons in the main SOL plasma (upstream of the divertor, along the field lines). Both Braginskii method of expansion in a small ratio of Coulomb collisional mean-free path $\lambda$ to the parallel plasma parameter variation scale length $L$ [4] and full kinetic Fokker-Planck modelling for small $\lambda / L$ ratios [5] predict that most of the parallel plasma heat conduction is carried by supra-thermal electrons. The velocities of such electrons are in the range of 3-5 of electron thermal velocity $v_{T_{e}}=\sqrt{T_{e} / m_{e}}$ (see [5,6], also [7], p.658), with the peak of the heat flux density in velocity space $v_{\|} v^{2} f_{e}\left(f_{e}\right.$ being the electron distribution function) located at electron kinetic energies $E_{e} \equiv m_{e} v^{2} / 2$ close to $6 \mathrm{~T}_{\mathrm{e}}$. Half of the total heat flux is carried by electrons with $E_{e} \geq 7 \mathrm{~T}_{\mathrm{e}}$ [5]. Such electrons can reach the target after only a few collisions and directly influence target plasma temperature, density and heat deposition profiles. They also raise the Debye sheath potential [5].

An increase in the potential drop at the target surface within the Debye sheath and magnetic pre-sheath (MPS) layers contributes to the build-up of the radial electric field, $\mathrm{E}_{\mathrm{r}}$, in the SOL. Since the largest discrepancies between SOLPS and the experimental target profiles were found for positions near the separatrix [1,2], one may expect the largest deviations between simulated and experimental $E_{r}$ profiles in the SOL also to occur closer to the separatrix position. This expectation is confirmed by the comparison between simulated and experimental $E_{r}$ profiles based on a number of SOLPS (B2.5-Eirene) and EDGE2D-Nimbus cases modelling AUG and JET plasmas, respectively, on the one hand, and experimental data from AUG, JET, JT-60U, TCV, on the other, presented in this paper.

In addition to discrepancies between simulated and experimental target $T_{e}$ and SOL $E_{r}$ profiles, there exists a long-standing issue of large discrepancies between modelled and measured Mach values of parallel ion flow in the SOL. 'Hotter' SOLPS solutions for the divertor, with higher target $T_{e}$, were found to predict larger Mach numbers and larger $E_{r}[8,3]$. It was suggested in the latter ref. that the discrepancies in target $T_{e}$ and Mach numbers can be related to each other, with the link between them being provided by the radial electric field in the SOL. Following [3], experimental confirmation for the existence of large $\mathrm{E}_{\mathrm{r}}$ in the SOL was obtained and numerical estimates for the relation between the $\mathrm{E}_{\mathrm{r}}$ and predicted parallel ion flows by SOLPS and EDGE2D were made. They lead to the conclusion that the correct, experimentally determined $\mathrm{E}_{\mathrm{r}}$ profiles in the SOL would indeed largely eliminate severe mismatches between field-dependent (dependent on the toroidal field direction, in single-null divertor configurations) modelled and experimental Mach numbers of the parallel ion flow in both AUG and JET [9].

This paper is organized as follows. Results of modelling $\mathrm{E}_{\mathrm{r}}$ in the SOL with the two widely used 2D fluid codes: SOLPS and EDGE2D-Nimbus, are presented in Section 2. They are followed by the results of the Lagmuir probe and Doppler reflectometer measurements of $E_{\mathrm{r}}$ in AUG described in Section 3, and the results of probe measurements in JET - in Section 4. 
Additional supporting experimental evidence from JT-60U and TCV is provided in Section 5. Implication of the discrepancies between modelled and experimental $E_{r}$ values, as well as high $E_{\mathrm{r}}$ values observed in some experiments, are discussed in Section 6. The work is summarised in Section 7.

\section{Modelling $E_{r}$ with SOLPS and EDGE2D}

The AUG and JET plasmas selected for the 2D edge modelling described here cover various conditions, primarily with respect to $\mathrm{n}_{\mathrm{e}}$ and $\mathrm{T}_{\mathrm{e}}$ values in the SOL and divertor. SOLPS cases were run with the coupled fluid plasma part, B2.5, and a kinetic Monte-Carlo neutral solver, Eirene. Similarly, EDGE2D was coupled with the neutral Monte-Carlo code Nimbus. Drift terms were fully switched on across the whole numerical mesh in the EDGE2D code. In B2.5, they were switched on across SOL, private region and about $1 / 3$ of the core region adjacent to the separatrix. For both SOLPS and EDGE2D results presented below, $T_{e}$ and plasma electric potential $\mathrm{V}_{\mathrm{p}}$ profiles across the SOL are plotted against distance from the separatrix at the outer midplane position.

The Ohmic SOLPS outer midplane profiles presented in Fig. 1 are based on the previously modelled standard AUG Ohmic case with medium density described in [10] (with fluid neutrals) and [2] (with Monte-Carlo neutrals). The normal Bt case (with the toroidal field $\mathrm{Bt}$ directed such that the ion $\nabla \mathrm{B}$ drift is towards the $\mathrm{X}$-point) analysed there matches very well experimental upstream (obtained near the outer midplane position) $\mathrm{n}_{\mathrm{e}}$ and $\mathrm{T}_{\mathrm{e}}$ profiles, with somewhat poorer match to the $T_{i}$ profile. It was not possible to match the divertor parameters in those calculations: the simulated outer target $T_{e}$ in the cases with Monte-Carlo neutrals was too low, and $n_{e}$ too high, compared to the experiment, and the radiated power (on deuterium only) slightly exceeded its experimental value, despite chemical sputtering being switched off, leading to almost complete elimination of impurities. Problems with matching target profiles are likely to be related with endemic deficiencies of the fluid codes in treating high energy electrons that are responsible for the bulk of the parallel heat flux. The AUG standard Ohmic case is quite representative of plasma edge conditions in this

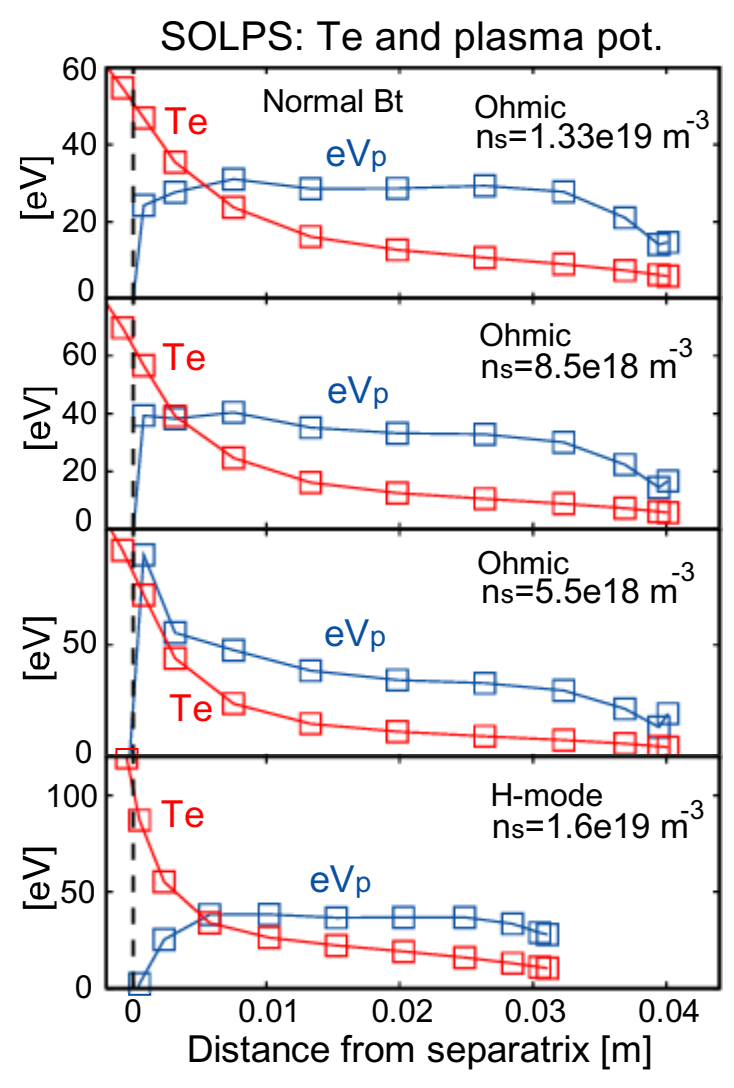

Fig. 1. Outer midplane profiles of electron temperature and plasma potential from SOLPS cases modelling AUG plasmas with normal Bt direction. Anomalous conductivity must have affected the $H$ mode case (see text for details). machine regarding collisionality. As was pointed out in [2], it also has the same midplane separatrix electron dimensionless collisionality (see the definition of it given by Eq. (1)) as that expected in ITER [11]. 
In the present study, two more cases, at lower electron separatrix densities $n_{s}$, were run, in order to cover possible regimes with 'hotter' (higher $\mathrm{T}_{\mathrm{e}}$ ) plasmas in the divertor. These simulated plasmas have no experimental equivalents. In addition, data for the low density Hmode shot in the normal Bt configuration, analysed earlier in [1], are also presented in Fig. 1.

SOLPS cases presented in Fig. 1 had small anomalous radial currents related to $\mathrm{E}_{\mathrm{r}}$ and $\nabla \mathrm{T}_{\mathrm{e}}$ (the latter - only for the H-mode case). The anomalous conductivity is controlled by the parameter fsig, and anomalous thermo-electric current - by falf. The electrical conductivity and thermo-electric coefficients are given by $f \operatorname{sig} \times 1.6 \times 10^{-19} n_{e}\left(m^{-3}\right)$ and falf $\times n_{e}\left(m^{-3}\right) T_{e}(e V)^{-1 / 2}$ is MKS units, respectively. The default values for both parameters in SOLPS are $1 \times 10^{-3}$. These anomalous currents were introduced into SOLPS for the sake of improving numerical stability of the code. For Ohmic cases shown in Fig. 1, fsig was reduced to $1 \times 10^{-4}$ and falf was set to zero. Such a change in the coefficients, compared to the cases with the default settings, was found to mainly influence (increase) electric potential on the first ring outside of the separatrix, while in the rest of the SOL $\mathrm{E}_{\mathrm{r}}$ was little affected. Further reduction in fsig didn't result in any appreciable changes in the potential profile, but detrimentally affected stability of the code runs. For the H-mode case, the default values for $f$ sig and falf were used, for the sake of numerical stability. All reversed field cases (with the toroidal field $\mathrm{Bt}$ directed such that the ion $\nabla \mathrm{B}$ drift is away from the $\mathrm{X}$-point), not shown in Fig. 1, also required default settings of these parameters.

As one can see from Fig. 1, for the highest density Ohmic and H-mode cases, plasma potential rises from the separatrix deeper into the SOL up to a distance of $\approx 0.5-1 \mathrm{~cm}$, then is stabilizes, and finally drops further out, at distances larger than $3 \mathrm{~cm}$. Too low, almost zero, potential on first ring outside of the separatrix in the H-mode case is an artifact related to anomalous radial currents introduced into SOLPS, as pointed out earlier. In the most of the $\mathrm{SOL}, \mathrm{E}_{\mathrm{r}}$ is almost zero for H-mode and the highest density Ohmic cases. For the Ohmic cases with reduced separatrix density of $8.5 \times 10^{-18} \mathrm{~m}^{-3}$, $\mathrm{E}_{\mathrm{r}}$ values obtained from the code were small, well below $-0.5 \nabla \mathrm{T}_{\mathrm{e}} / \mathrm{e}$, across most of the SOL. They only showed an increase in the far SOL, at the position of $\approx 3.25 \mathrm{~cm}$ from the separatrix, where the plasma density is already too small. The only case that had appreciable $E_{\mathrm{r}}$ values across most of the SOL was the Ohmic case with extremely low $\mathrm{n}_{\mathrm{s}}=5.5 \times 10^{18} \mathrm{~m}^{-3}$. The $-\mathrm{eE}_{\mathrm{r}} / \nabla \mathrm{T}_{\mathrm{e}}$ ratio reached $\approx 1$ near the separatrix in this case. The main reason for the $-\mathrm{eE}_{\mathrm{r}} / \nabla \mathrm{T}_{\mathrm{e}}$ increase at low densities is the peaking of target $T_{e}$ profiles. This results in the peaking of the target Debye sheath potential profiles that raises the upstream $E_{r}$ (see Sec. 6).

The selected EDGE2D cases for Ohmic JET shots repeat those previously modelled and described in [12]: \#56723 for normal, and \#59737 - for reversed Bt cases (note different meshes in Fig. 2). Two density phases of these shots, referred to as having 'high' and 'low' separatrix density $n_{s}$, were modelled in [12] by varying the amount of the gas puff in the modelling. Contrary to SOLPS cases, which were severely constrained by having to match extremely good quality upstream profiles, and consequently having problems with matching target profiles, the latter were given much more weight in specifying input parameters for EDGE2D runs. Experimental target profiles, mainly of the $n_{e}$ and $T_{e}$, obtained by Langmuir probes, were fairly well matched in the EDGE2D modelling.

In the original EDGE2D cases described in [12], drifts were switched on only in the SOL, but switched off in the core, owing to numerical instabilities originating in the core region. 
Recent improvements in the code have enabled switching drifts on everywhere in the grid. In the present simulations, these cases were run further with drifts switched on everywhere up to the new steady-state, which however showed only insignificant deviations in midplane $T_{e}$ and $\mathrm{V}_{\mathrm{p}}$ profiles in the SOL, as well as in the target parameters, from the original cases. However, Mach numbers of the ion parallel flow were somewhat higher. Similar to SOLPS, small radial anomalous conductivity is used in EDGE2D cases, but only in the core region. In contrast to SOLPS, there are no anomalous currents in the SOL and divertor regions.

The EDGE2D H-mode case with normal Bt was modelled in [13]. It corresponds to JET Type-I ELMy H-mode discharge with NBI input power of $12 \mathrm{MW}, \mathrm{Bt}=2.4 \mathrm{~T}$ and $\mathrm{Ip}=2.5 \mathrm{MA}$. Drifts were switched on in these calculations. In the present paper, the same case was continued with the reversed field direction until it reached steadystate.

The main signatures of the $\mathrm{V}_{\mathrm{p}}$ profiles shown in Fig. 2 repeat those of the SOLPS cases. Some difference between the normal and reversed field $\mathrm{V}_{\mathrm{p}}$ profiles for low density Ohmic cases may be caused by lower, by $\approx 10 \%$, density in reversed $\mathrm{Bt}$ case, which also gives rise to higher separatrix $\mathrm{T}_{\mathrm{e}}$. (The same difference between high density Ohmic cases, however, doesn't result in any appreciable difference in $V_{p}$ profiles). Similarly to SOLPS cases, the $\mathrm{V}_{\mathrm{p}}$ profiles across most of the SOL show almost no resemblance to the $T_{e}$ profiles. Steepening of $V_{p}$ profiles for low density cases is mainly caused by peaking of target $T_{e}$ profiles, similar to SOLPS cases.

Since kinetic effects in the parallel electron

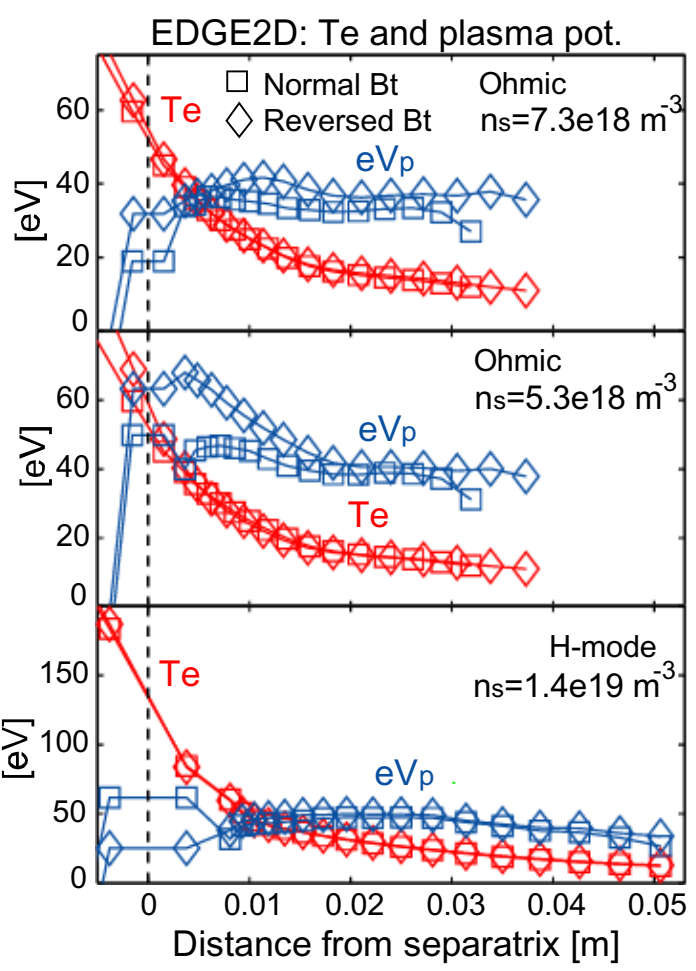

Fig. 2. Outer midplane profiles of electron temperature and plasma potential from EDGE2D cases modelling JET plasmas.

transport have been invoked earlier in order to explain the discrepancy between measured and simulated parameters in the divertor of AUG, it is useful to introduce the dimensionless electron collisionality upstream, close to the outer midplane. For a rough estimate, it can be defined, following [7] (p.194, Eq. (4.105)) as:

$$
v_{e e}^{*}=10^{-16} \frac{n_{e}\left(m^{-3}\right) \pi q_{95} R(m)}{T_{e}(e V)^{2}},
$$

where the connection length $L$ was replaced by $\pi q_{95} R$, with $q_{95}$ being the safety value at the $95 \%$ poloidal flux surface. This collisionality, calculated for separatrix $n_{e}$ and $T_{e}$ values, will be used to compare different code runs and different experiments/plasmas. For SOLPS cases shown in Fig. 1, collisionalities $v_{e e}^{*}$ are (from top to the bottom box): 14.1, 8.4, 6.6 and 3.5. As pointed out earlier, the highest collisionality, corresponding to the standard AUG Ohmic plasma, is very close to the expected collisionality in ITER. For the EDGE2D cases presented in Fig. 2, the collisionalities are (from top to bottom): 7.2, 6.3, 1.1. Together, SOLPS and EDGE2D cases cover a fairly broad range of upstream separatrix collisionalities. These 
values will be compared with the estimated collisionalities in the experiments discussed below.

Experimental data on the radial electric field in the SOL presented below by no means represent a thorough review on this subject. A rather limited selection of experimental devices and results of the $E_{r}$ measurements mainly with Langmuir probes covered in the following sections is only meant to demonstrate the most basic features of the $E_{\mathrm{r}}$ profiles in the SOL. However, even a brief overview of the available probe data from AUG and JET, as well as from JT-60U, TCV and earlier published Alcator C-Mod data, provides a convincing evidence for large discrepancies between measured and modelled (with $2 \mathrm{D}$ fluid codes) $\mathrm{E}_{\mathrm{r}}$ profiles.

\section{Experimental data on $E_{r}$ from AUG}

Reciprocating Langmuir probes, which are typically used to evaluate plasma potential $\mathrm{V}_{\mathrm{p}}$, directly measure floating potential $\mathrm{V}_{\mathrm{f}}$, among other plasma parameters (mainly $\mathrm{T}_{\mathrm{e}}$ and the ion saturation current $I_{\text {sat }}$, from which plasma density can be evaluated). Evaluation of the $E_{r}$ therefore requires knowledge of the difference $\left(V_{p}-V_{f}\right)$ which is usually taken from theory to be $\sim 3 \mathrm{~T}_{\mathrm{e}} / \mathrm{e}$ (see below). In order to provide consistency between various pieces of experimental information supplied by different machines on the evaluated $E_{r}$, the most frequently encountered assumption $\mathrm{e}\left(\mathrm{V}_{\mathrm{p}}-\mathrm{V}_{\mathrm{f}}\right) / \mathrm{T}_{\mathrm{e}}=3$ will be used throughout this paper. Thus, by differentiating the equation $\mathrm{V}_{\mathrm{p}}=\mathrm{V}_{\mathrm{f}}+3 \mathrm{~T}_{\mathrm{e}} / \mathrm{e}$ (see e.g. [7], p.520, Sec. 17.3), the radial electric field will be derived as:

$$
E_{r}=-\nabla V_{f}-3 \nabla T_{e} / e
$$

from probe measurements of $V_{f}$ and $T_{e}$, where the gradient sign implies gradient over the perpendicular (radial) direction.

Figure 3 shows $T_{e}$ and $V_{f}$ data obtained by the reciprocating Mach probe (Langmuir probe capable of measuring Mach number of parallel ion flow) introduced just above the outer midplane, as described in $[14,15]$. The discharge parameters were: line-average density $\bar{n}_{e}=3.65 \times 10^{19} \mathrm{~m}^{-3}$, toroidal field $\mathrm{Bt}=2 \mathrm{~T}$, plasma current $\mathrm{Ip}=0.8 \mathrm{MA}, q_{95}=4$. This is a standard AUG Ohmic shot, with the same parameters as shot \#18737 modelled by SOLPS and described in Section 2. The data presented here were obtained by the same pins of the reciprocating probe as those used for measuring parallel ion Mach number. The probes, separated by a partition, were facing outer ('l-values') or inner ('r-values') divertor along the field lines. Owing to a strong parallel ion flow in the direction from the outer to inner divertor at the probe position in this normal Bt discharge, plasma density (ion saturation current was the directly measured signal) was larger at the flow-facing pins ('l-values'), leading also to higher floating potential than for downstream pins, as one can see from Fig. 3. The difference between the $V_{f}$ values is especially large near the separatrix. Calculated plasma potentials show steep rise towards the separatrix position for both up- and down-stream pins, with the calculated $-\mathrm{eE}_{\mathrm{r}} / \nabla \mathrm{T}_{\mathrm{e}}$ values obtained as $\mathrm{e} \Delta \mathrm{V}_{\mathrm{f}} / \Delta \mathrm{T}_{\mathrm{e}}$ over the last $1.5 \mathrm{~cm}$ of the probe reciprocation being of order 3 . This is in a sharp contrast with the modelling results described in Sec. 2, where $E_{r}$ is nearly zero for most of the SOL (see the $V_{p}$ profile in the top box of Fig. 1). Due to low $T_{e}$ values, $\approx 18 \mathrm{eV}$, measured by the probe even at the closest position to the separatrix, the probe apparently didn't go far into the plasma, stopping at a distance of 0.5 
$-1 \mathrm{~cm}$ from the separatrix, judging by the value of the separatrix $\mathrm{T}_{\mathrm{e}}(\approx 45 \mathrm{eV}$, according to the SOLPS modelling) and the $\mathrm{T}_{\mathrm{e}}$ profile measured by the YAG laser.
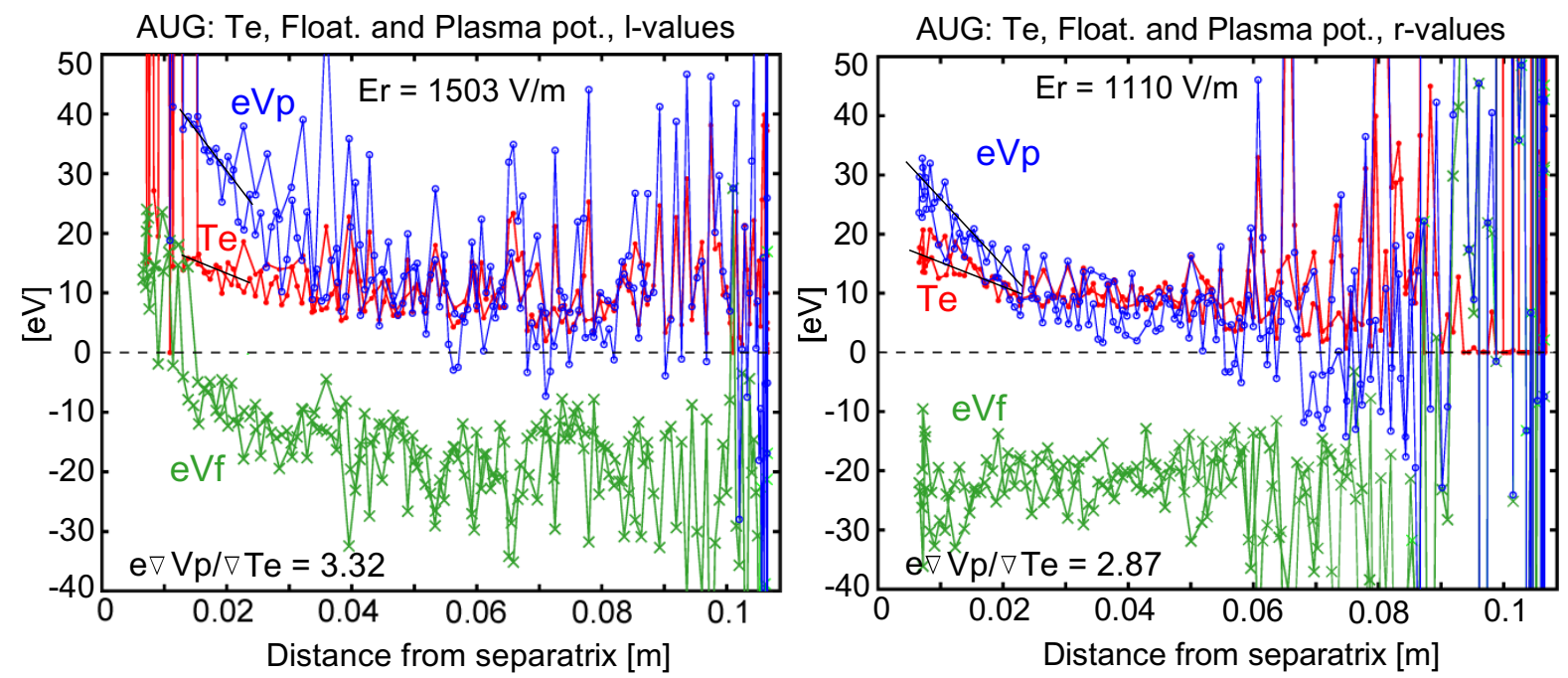

Fig. 3. $T_{e}$, floating and plasma potential profiles invoked from the reciprocating Langmuir probe in the standard AUG Ohmic shot. ' $l$ ' and ' $r$ '-values refer to data coming from the probes separated by a partition and facing outer and inner divertor along the field lines, respectively. 'Distance from the separatrix' is measured along the probe reciprocation trajectory, which is above the midplane.

The average electron density (between the two probe pins, with the data from the r-pin being linearly extrapolated onto the innermost position of the 1-pin) was $1.6 \times 10^{19} \mathrm{~m}^{-3}$. Taking this density, $\mathrm{T}_{\mathrm{e}}=18 \mathrm{eV}$, machine parameters and $\mathrm{q}_{95}=4$, one obtains for the collisionality defined according to Eq. (1): $v_{e e}^{*}=105$, which is the highest value of all measured and code simulated cases presented in this paper. Note however, that this collisionality is calculated not at the separatrix position but at a position inside of the scrape-off layer. Also, there are doubts related to the correctness of the determination of electron temperature from the Langmuir probes, since the probe $T_{e}$ profile is flatter than that obtained from the YAG laser measurements (see below in this section). Related to a possible underestimate of the probe $T_{e}$, is the apparent overestimate of its density $\mathrm{n}_{\mathrm{e}}\left(1.6 \times 10^{19} \mathrm{~m}^{-3}\right)$ which is higher than the electron separatrix density $\mathrm{n}_{\mathrm{s}}=1.33 \times 10^{19} \mathrm{~m}^{-3}$ of the SOLPS solution (see Fig. 1, top box).

An estimate of the $E_{\mathrm{r}}$ in the SOL can also be obtained from Doppler reflectometry. The technique and diagnostic on AUG are described in detail in [16]. By poloidally tilting a microwave reflectometer a Doppler frequency shift is induced in the measured turbulence spectrum $f_{D}=u_{\perp} k_{\perp} / 2 \pi$ which is directly proportional to the velocity $u_{\perp}=v_{E \times B}+v_{p h}$ (where $\mathrm{v}_{\mathrm{ph}}$ is phase velocity) of the turbulence moving in the plasma $\mathrm{E}_{\mathrm{r}} \times \mathrm{B}$ frame. $\mathrm{k}_{\perp}$ is the measured turbulence wavenumber selected by the tilt and plasma geometry. Linear and non-linear numerical turbulence simulations indicate that turbulence phase velocity $\mathrm{v}_{\mathrm{ph}}$ is of the order of a few tens to hundreds of $\mathrm{m} / \mathrm{s}$ at the measured $\mathrm{k}_{\perp} \sim 8 \mathrm{~cm}^{-1}[17,18]$, which is small compared to $v_{E \times B}$ thus allowing $E_{r}$ to be approximated directly as $u_{\perp} B$. Although the electron diamagnetic velocity is a factor of 3 or so larger than $u_{\perp}$ (see below, the combined effect of electron density and temperature gradients measured by the YAG laser in Fig. 4), a $\mathrm{v}_{\mathrm{ph}} \sim 0$ is not unexpected since linear gyrokinetic simulations on closed field lines indicate that the drives from the comparable density and ion temperature gradient lengths can counteract [19]. 
Note that the sharp reversal of $E_{r}$ across the separatrix reflects the strong velocity shear in the pedestal gradient region (see e.g. [16]).

Fig. 4. Experimental and modelled profiles for the Ohmic AUG shot: Er profile measured by the Doppler reflectometer, $-\nabla T_{e} / e$ and $-T_{e} \nabla n_{e} / n_{e} e$ profiles from the YAG laser, averaged $E_{r}$ from the reciprocating Langmuir probe (see original data in Fig. 3), and $E_{r}$ from Ohmic SOLPS cases with two levels of density (see $V_{p}$ profiles in Fig. 1).

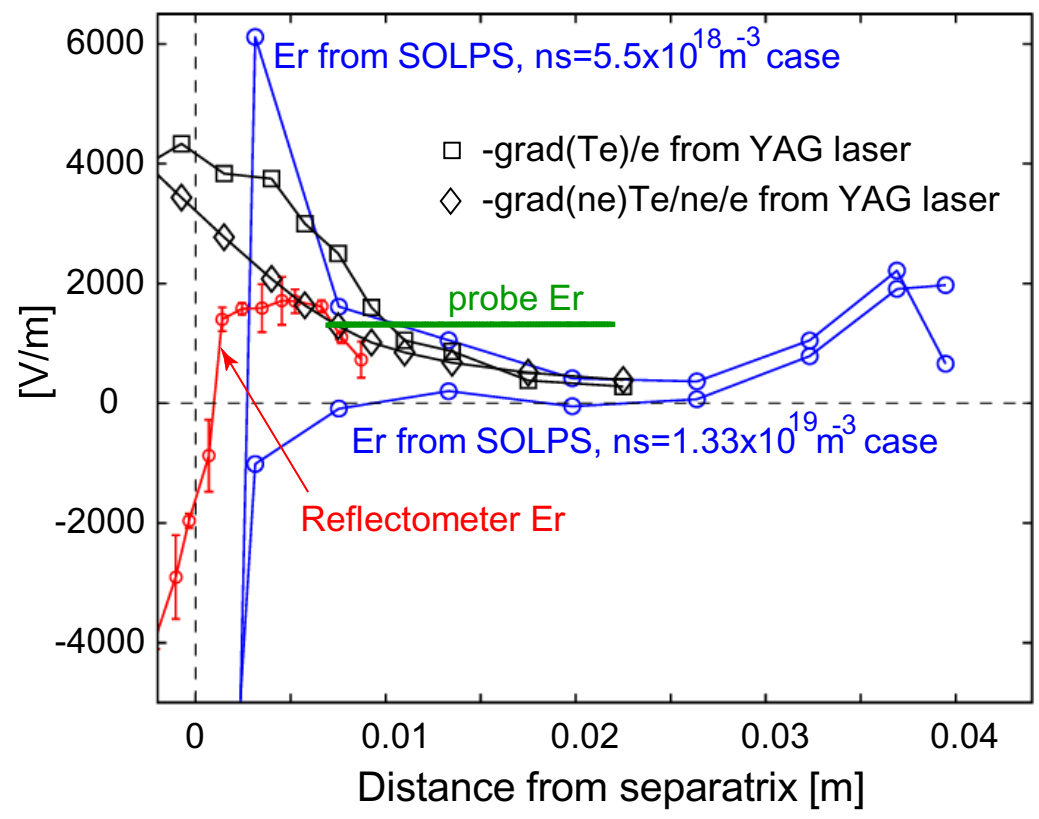

Fig. 4 shows the measured $E_{r}$ profile (assuming $v_{p h}=0$ ) for the equivalent Ohmic shot \#18813 from inside the separatrix to a few $\mathrm{cm}$ into the SOL. The radial extent is determined by the reflectometer probing frequency range and the SOL density profile. Also shown in this figure are $-\nabla \mathrm{T}_{\mathrm{e}} / \mathrm{e}$ and $-\mathrm{T}_{\mathrm{e}} \nabla \mathrm{n}_{\mathrm{e}} / \mathrm{n}_{\mathrm{e}} \mathrm{e}$ profiles obtained from the interpolation of the YAG laser data, averaged $E_{r}$ obtained from the data shown in Fig. 3 (the data is averaged over the radial distance of $1.5 \mathrm{~cm}$ as well as between the two probes), and the simulated $\mathrm{E}_{\mathrm{r}}$ from the two Ohmic SOLPS cases for the highest and lowest density using $V_{p}$ profiles shown in Fig. 1. Outer midplane YAG laser $n_{e}$ and $T_{e}$ profiles, as well as the $T_{i}$ profile measured by the Li beam diagnostic, can be found in [2].

As can be seen from Fig. 4, the Doppler reflectometer $E_{r}$ is below $-\nabla T_{e} / e$, in contrast with the ratio $-\mathrm{eE}_{\mathrm{r}} / \nabla \mathrm{T}_{\mathrm{e}} \sim 3$ following from the probe measurements. There is, on the other hand, no direct conflict with the $E_{\mathrm{r}}$ invoked from the probe. The average probe $E_{\mathrm{r}}$ value, $1307 \mathrm{~V} / \mathrm{m}$, is quite close to the reflectometer data, albeit at larger radii. An additional complication with the data for this shot comes from the difference in the gradients of $T_{e}$ profiles measured by the YAG laser and the Langmuir probe, as pointed out above. The YAG $T_{e}$ profile is fairly well resolved spatially, and it exhibits sharp flattening of the $T_{e}$ profile over the distance indicated by the segment labeled 'probe $\mathrm{E}_{\mathrm{r}}$ ' in Fig. 4. The $\nabla \mathrm{T}_{\mathrm{e}}$ from YAG changes from being 7 times larger than the averaged probe $-\mathrm{eE}_{\mathrm{r}}$ at the innermost probe position (where the probe $\mathrm{T}_{\mathrm{e}}$ coincides with the $Y A G T_{e}$, both equal to $\approx 18 \mathrm{eV}$; this equality is not coincidental, but was used to determine the absolute probe position with respect to that of YAG chords, as plotted in Fig. 4) to being somewhat smaller than it at the end of the 'probe $E_{r}$ ' segment. Provided one uses the YAG $T_{e}$ profile, probe floating potential averaged between the two pins and $-3 \mathrm{~T}_{\mathrm{e}} / \mathrm{e}$ for the difference between $\mathrm{V}_{\mathrm{f}}$ and plasma potential $\mathrm{V}_{\mathrm{p}}$, one obtains high $\mathrm{E}_{\mathrm{r}}$ values, $\approx$ $8000 \mathrm{~V} / \mathrm{m}$, at the innermost probe position, and only $\approx 800 \mathrm{~V} / \mathrm{m}$ for the outermost position of the 'probe $\mathrm{E}_{\mathrm{r}}$ ' segment. 
SOLPS $E_{r}$ profile for the higher density Ohmic case, that matches experimental upstream profiles shown in Fig. 4, is much below both the reflectometer and Langmuir probe values, except for the outer SOL positions $>3 \mathrm{~cm}$ away from the separatrix. It is possible to greatly increase $E_{r}$ by a large reduction in the separatrix density. Such SOLPS solutions, however, are unrealistic, since their separatrix $n_{e}$ and $T_{e}$ values strongly deviate from the relation between these two quantities established from the YAG laser data.

\section{Experimental data on $E_{r}$ from JET}

Fig. 5. Relationship between electron temperature $T_{e}$ and floating potential $V_{f}$ obtained by the reciprocating Langmuir probe for a number of JET discharges with different confinement properties and taken at different positions during the probe reciprocation. The floating potential is measured with respect to the torus potential The figure is replicated from ref. [12], with minor alterations.

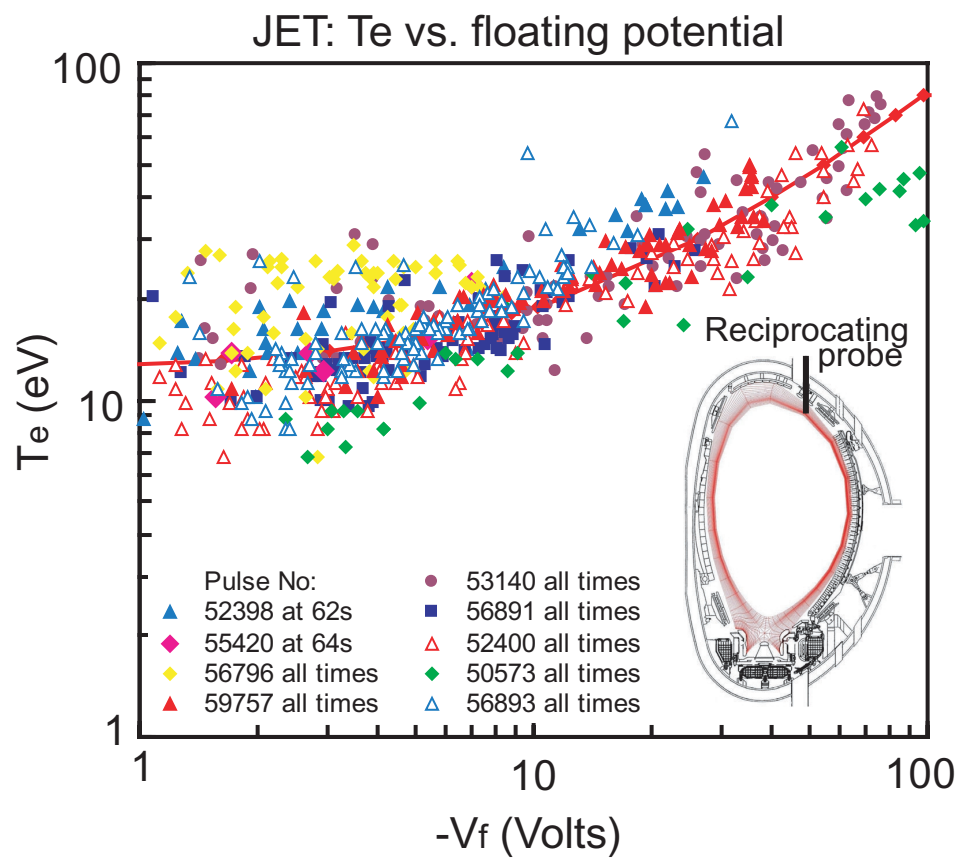

The largest statistical information on the distribution of plasma potential in the SOL was obtained on JET. The results are described in the paper dedicated to comparison between experimental and code simulated parallel ion flows [12]. The reciprocating probe was introduced from the top of the machine, but shifted outward, as indicated in Fig. 5. A number of representative Ohmic, L- and H-mode shots was used to compile the database on the relationship between $T_{e}$ and $V_{f}$, the latter measured with respect to the torus potential (the same as the target potential). The results are shown in Fig. 5. The $\mathrm{T}_{\mathrm{e}}$ values were obtained by averaging between the two probe pins facing opposite sides (inner and outer target, along the field lines) of the Mach probe, whereas the $V_{\mathrm{f}}$ data were taken by a separate probe, protruding into the plasma (a small adjustment has been made in Fig. 5 for different positions of $T_{e}$ and $\mathrm{V}_{\mathrm{f}}$ with respect to the plasma position). The dependence of $\mathrm{V}_{\mathrm{f}}$ on $\mathrm{T}_{\mathrm{e}}$ can be interpolated by the offset linear dependence (in $\mathrm{eV}$ ): $\mathrm{eV}_{\mathrm{f}}=-1.43 \mathrm{~T}_{\mathrm{e}}+17$, and the best fit to the experimental data is shown by the solid line in Fig. 5. This fit is used here to evaluate plasma potential $V_{p}$. A relation between $V_{f}$ and $V_{p}$ can be obtained by using a formula given by Stangeby [20]. Combining contributions from the Debye sheath (Eq. (16) of [20]) and magnetic pre-sheath (formula on p.686 of [20]) one obtains:

$e\left(V_{f}-V_{p}\right)=0.5 T_{e} \ln \left[2 \pi \frac{m_{e}}{m_{i}} \frac{\left(1+T_{i} / T_{e}\right)}{(1-\delta)^{2}}\right]-0.7 T_{e}$ 
where $\delta$ is secondary electron emission. For $\mathrm{T}_{\mathrm{i}} / \mathrm{T}_{\mathrm{e}}=2$, which seems in agreement with experimental data, deuterium plasma and $\delta=0.3$, one obtains $\mathrm{e}\left(\mathrm{V}_{\mathrm{f}}-\mathrm{V}_{\mathrm{p}}\right)=2.98 \mathrm{~T}_{\mathrm{e}}$, which is in a good agreement with Eq. (2).

Combining Eq. (2) with the best fit for the $\mathrm{V}_{\mathrm{f}}$ versus $\mathrm{T}_{\mathrm{e}}$ dependence, one obtains: $-\mathrm{eE}_{\mathrm{r}} / \nabla \mathrm{T}_{\mathrm{e}}=$ 1.57. This ratio can be reduced below 1.57 by increasing secondary emission, with $\delta>0.3$, or by increasing $\mathrm{T}_{\mathrm{i}} / \mathrm{T}_{\mathrm{e}}$ ratio, above 2 . It can, on the other hand, also be increased if one takes into account the presence of impurities (formulas above assume pure deuterium plasma). The ratio $-\mathrm{eE}_{\mathrm{r}} / \nabla \mathrm{T}_{\mathrm{e}}$ invoked from the JET data, despite being below that found in AUG, is still in a clear contradiction with the results of the EDGE2D modelling described in the previous section. Experimental trends observed in AUG and JET are confirmed by the data supplied from JT-60U and TCV tokamaks considered next.

\section{Supporting experimental evidence from JT-60U and TCV}

Reciprocating probe measurements on JT-60U described in this section were performed by a double-sided (Mach) probe introduced from the low field side, $35 \mathrm{~cm}$ below the equatorial midplane. Given the size of the machine $(\mathrm{R}=3.44 \mathrm{~m}, \mathrm{a}=0.96 \mathrm{~m})$, the probe position may be regarded as being very close to the outer midplane, similar to the geometry of AUG measurements. The measured $V_{f}$ and $T_{e}$ profiles shown in Fig. 6 are for the L-mode shot in

Fig. 6. Te, floating and plasma potentials for the L-mode JT-60U shot measured by the reciprocating Langmuir probe introduced below the outer midplane position. See details in the text.

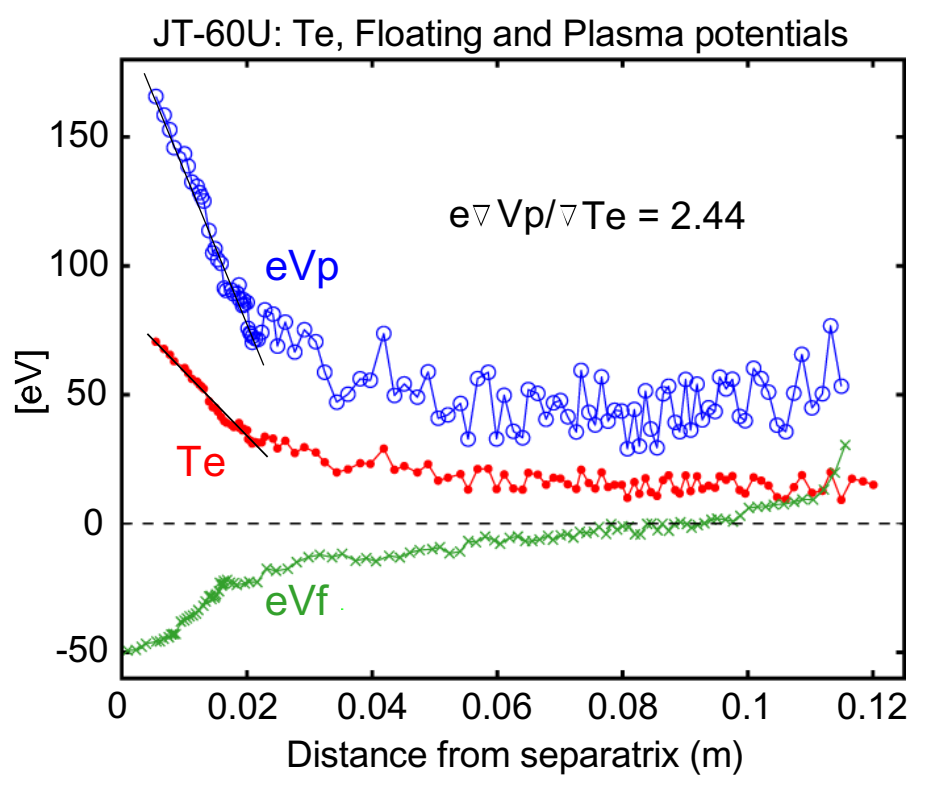

normal Bt configuration, with $\mathrm{Bt}=3.5 \mathrm{~T}, \mathrm{Ip}=1.7 \mathrm{MA}$, NBI power of $\approx 4.2 \mathrm{MW}$ and line average density $\overline{\mathrm{n}}_{\mathrm{e}}=1.5 \times 10^{19} \mathrm{~m}^{-3}$. This shot was part of the density scan with $\overline{\mathrm{n}}_{\mathrm{e}}$ varying from 1.0 to $3.2 \times 10^{19} \mathrm{~m}^{-3}$, and was selected as representative for the analysis of the nature of parallel ion flows in [21] (see Fig. 5 and related text in this ref., where details of the measurements can also be found). The $T_{e}$ plotted in the figure is an averaged value of temperatures measured by the two sides of the Mach probe, while the $V_{f}$ was measured with a separate probe protruding into the plasma and being $4.5 \mathrm{~mm}$ closer to the separatrix than the other probes used to measure $n_{e}$ and $T_{e}$. The $T_{e}$ data in Fig. 6 were correspondingly shifted from the $V_{f}$ data by this distance. As one can see from this figure, the $V_{p}$ profile within the 
first $2 \mathrm{~cm}$ into the $\mathrm{SOL}$ is rather steep, with the $-\mathrm{eE}_{\mathrm{r}} / \nabla \mathrm{T}_{\mathrm{e}}$ ratio being 2.44 . The average electron density (between the two probe pins) reached $6.5 \times 10^{18} \mathrm{~m}^{-3}$. Taking this density and the highest $\mathrm{T}_{\mathrm{e}}(71 \mathrm{eV})$ measured by the probes, machine parameters and $\mathrm{q}_{95}=3.5$, one obtains for the collisionality defined according to Eq. (1): $v_{e e}^{*}=4.9$, which is in between collisionalities estimated for the AUG H-mode case and the Ohmic case with $\mathrm{n}_{\mathrm{s}}=5.5 \times 10^{18}$ $\mathrm{m}^{-3}$.

Langmuir probe measurements in TCV [22] are in good agreement with basic signatures of the $V_{p}$ profiles observed in AUG and JT-60U. Fig. 7 shows $V_{p}$ and $T_{e}$ profiles obtained in this machine for an Ohmic plasma with $\mathrm{Bt}=1.43 \mathrm{~T}$, $\mathrm{Ip}=260 \mathrm{kA}$, in reversed Bt configuration. The plasma, which is usually formed in the top half of the vacuum chamber in TCV, was positioned near the centre of the machine in these experiments, so that the reciprocating Mach probe, introduced from the outer (low field) side, as in AUG and JT-60U cases, could reach the separatrix. Similar $T_{e}$ but slightly different $V_{f}$ profiles were obtained for the three positions of the plasma, with the axis of the magnetic configuration coinciding with the equatorial plane of the vacuum chamber (in which case the probe movement was exactly within the midplane; $Z=0 \mathrm{~cm}$, the case shown in Fig. 7), as well as slightly upward $(Z=+10$ $\mathrm{cm})$ and downward $(Z=-10 \mathrm{~cm})$ shifted plasmas. The three pins of the probe, used to measure $T_{e}, n_{e}$ and $V_{f}$, were protruding into the plasma and on the same magnetic flux surface.

Fig. 7. Te, floating and plasma potentials for the Ohmic TCV shot measured by the reciprocating Langmuir probe introduced along the outer midplane. See details in the text.

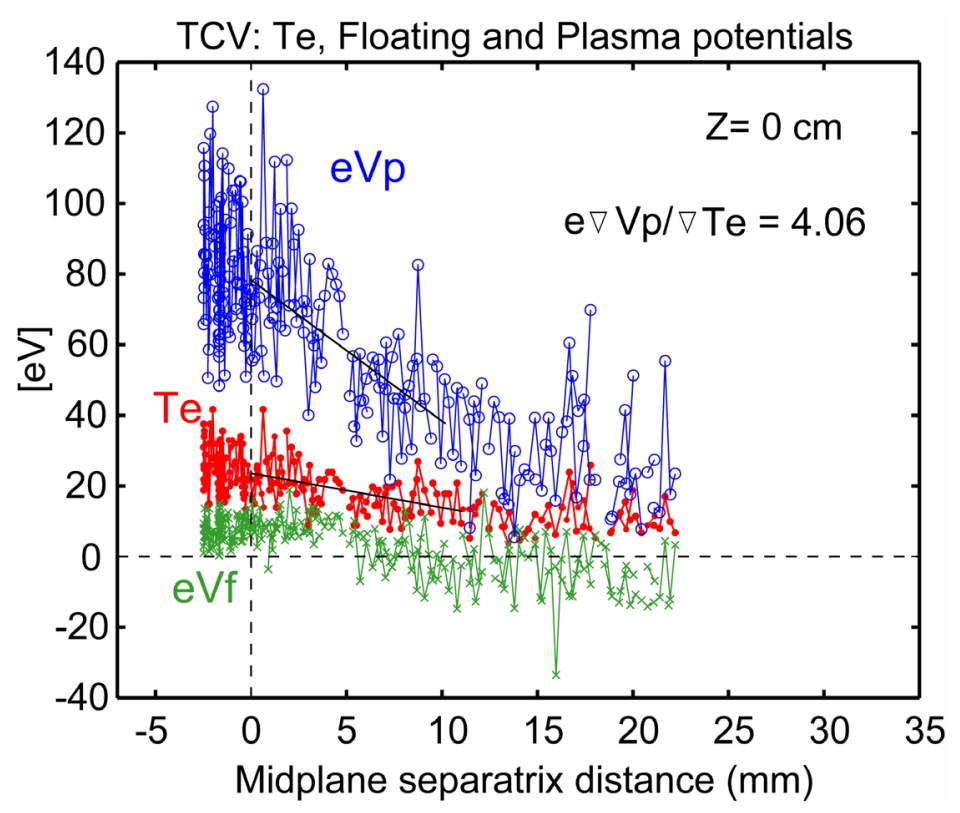

As one can see from Fig. 7 , the $-\mathrm{eE}_{\mathrm{r}} / \nabla \mathrm{T}_{\mathrm{e}}$ ratio in $\mathrm{TCV}$ for this configuration, using probe positions within $1 \mathrm{~cm}$ from the separatrix, is even larger than in AUG and JT-60U plasmas, being $\approx 4$ (for $Z=+10 \mathrm{~cm}$ configuration this ratio was 3.27 , and for $Z=-10 \mathrm{~cm}-4.97$ ). The line-averaged plasma density was $\overline{\mathrm{n}}_{\mathrm{e}}=4.2 \times 10^{19} \mathrm{~m}^{-3}$, in the middle of the density range, from 1.7 to $7.3 \times 10^{19} \mathrm{~m}^{-3}$, in this series of discharges aimed mainly at measuring Mach numbers of the parallel ion flow. Using measured separatrix parameters: $n_{s}=1.4 \times 10^{19} \mathrm{~m}^{-3}, T_{e}=25 \mathrm{eV}$, the machine parameter $\mathrm{R}=0.87 \mathrm{~m}\left(\mathrm{a}=0.25 \mathrm{~cm}\right.$ for these plasmas) and the safety value $\mathrm{q}_{95}=$ 3.5 , one obtains for the dimensionless collisionality at the separatrix: $v_{e e}^{*}=21.6$, which is the 
second highest value (after the AUG collisionality, calculated for a position outside of the separatrix) of all measured and code simulated cases presented in this paper. It has to be noted, however, that the TCV tokamak has highly unconventional divertor geometry with a very long outer divertor leg. Estimates show that this should increase the effective dimensionless collisionality by more than a factor of 2 .

\section{Discussion}

Comparison between measured and simulated radial electric fields in the SOL reveals a clear gap between experimental and modelled $\mathrm{E}_{\mathrm{r}}$ values, despite both experimental and modelled plasmas covered wide and overlapping range of experimental conditions with respect to the dimensionless separatrix collisionalitiy. The $-\mathrm{eE}_{\mathrm{r}} / \nabla \mathrm{T}_{\mathrm{e}}$ ratios at the outer midplane in the code simulations are typically well below 0.5 . In contrast, in all experimental Langmuir probe profiles presented in this paper, as well as statistical results from JET, this ratio is above 1.5. Earlier experimental evidence from Alcator C-Mod [23] also suggests large and positive $-\mathrm{eE}_{\mathrm{r}} / \nabla \mathrm{T}_{\mathrm{e}}:$ ratios $\approx 1.8$ and 1.7 follow from 4 data points for $\mathrm{V}_{\mathrm{f}}$ and $\mathrm{T}_{\mathrm{e}}$ outside of limiter shadows, averaged between the two probe pins (facing inner and outer divertors), for lower single-null (normal Bt direction) and upper single-null (reversed Bt direction) configurations, respectively. The reciprocating probe was introduced from the outer (low field) side just above the equatorial plane. Dimensionless collisionalities $v_{e e}^{*}$ calculated according to Eq. (1) were $\approx 16$ and 12 , for lower and upper single-null configurations, respectively, being similar to those for the modelled standard AUG Ohmic case. Data from the probe introduced from the inner (high field) side, however, were more complex, with $\mathrm{E}_{\mathrm{r}}$ extracted from the first two probe positions (deepest in the plasma) being negative, but for positions further out positive, with the $-\mathrm{eE}_{\mathrm{r}} / \nabla \mathrm{T}_{\mathrm{e}}$ ratio similar to those from the outside probe. Electron temperatures measured by the inside probe were substantially lower than those measured by the outside probe.

Low $E_{\mathrm{r}}$ values following from the code results presented here are a typical feature of solutions where neutrals are described by kinetic Monte-Carlo codes (Eirene and Nimbus in SOLPS and EDGE2D, respectively). In contrast, SOLPS cases with fluid neutrals exhibit higher $\mathrm{E}_{\mathrm{r}}$ values and positive throughout most of the SOL, but with the $-\mathrm{eE}_{\mathrm{r}} / \nabla \mathrm{T}_{\mathrm{e}}$ ratios still well below 1 for the standard AUG Ohmic case. Examples of SOLPS solutions with fluid neutrals and positive $\mathrm{E}_{\mathrm{r}}$ in the SOL can be found in [10] and [24]. The difference between cases with fluid and Monte-Carlo neutrals may be related to a much higher energy of neutrals in fluid cases, resulting in 'hot' (with high $\mathrm{T}_{\mathrm{e}}$ ) solutions in the divertor. However, as was pointed out earlier, even substantial variation of neutrals' behaviour in Eirene has not led to sufficiently 'hot' divertor solutions, that would be consistent with experiment. An issue of Monte-Carlo versus fluid neutral models and their impact on SOLPS solutions and $\mathrm{E}_{\mathrm{r}}$ values will be thoroughly dealt with in the near future.

The present assumption is that the discrepancies between experimental and simulated $\mathrm{E}_{\mathrm{r}}$ values should be attributed to the effect of supra-thermal electrons originating from the main SOL, upstream of the divertor. (No direct link between the $-\mathrm{eE}_{\mathrm{r}} / \nabla \mathrm{T}_{\mathrm{e}}$ ratios and collisionalities $v_{e e}^{*}$ can however be established from the analysis of experimental data presented here, although such a link is clearly seen in the SOLPS cases for the same magnetic configuration (lower density cases have larger $-\mathrm{eE}_{\mathrm{r}} / \nabla \mathrm{T}_{\mathrm{e}}$ )). Unfortunately, to our knowledge, there is no direct experimental evidence confirming the existence of a significant population of energetic 
electrons. Indirectly, in a number of publications (see e.g. [25] and refs. therein) it has been concluded that in medium density plasmas target probes read considerably higher $T_{e}$ than the true local value due to the contribution of weakly collisional electrons originating in regions of higher temperatures further upstream.

So far, the strongest evidence for the existence of large population of supra-thermal electrons comes from kinetic modelling. In [5], a situation with a much higher upstream than downstream $T_{e}$ and the ratio of Coulomb collisional mean-free path $\lambda$ to the parallel plasma parameter variation scale length $L$ of 0.1 (at the 'hot' end) was modelled with the FokkerPlanck kinetic code ALLA. Near the 'cold' boundary the electron heat flux was found to be totally dominated by tail electrons coming from hotter regions upstream, with the heat flux density being relatively constant (varying by not more than $\approx 50 \%)$ in the $\mathrm{E}_{\mathrm{e}} / \mathrm{T}_{\mathrm{e}}$ range $\left(\mathrm{E}_{\mathrm{e}}\right.$ electron kinetic energy) from 3 to 15 , with $\mathrm{T}_{\mathrm{e}}$ being local ('cold') electron temperature. At the same time, the 'hot' boundary was under-populated by hot electrons. This situation is very similar to that encountered in typical AUG plasmas [2] and also expected in ITER [5,2], with $\mathrm{T}_{\mathrm{e}}$ varying strongly along the field lines and similar upstream collisionality levels. For example, for the SOLPS case simulating the second, higher density phase of the standard Ohmic AUG shot analysed in [2] and discussed in Sec. 2, the effective separatrix collisionality $v_{e e}^{*}$ at the outer midplane position determined according to Eq. (1) is equal to 14.1. This value is not far from the $L / \lambda$ ratio of 10 used in the kinetic modelling, to which it can be directly compared. Since $v_{e e}^{*}$ reflects the collisionality of thermal electrons, suprathermal electrons from the $\sim 4^{\text {th }}$ tail of the Maxwellian distribution (in velocity space) will have the effective $L / \lambda$ ratio of $\sim 0.1$, for the nominal (that is, calculated for thermal speeds) $L / \lambda$ ratio of 10 , owing to the particle velocity scaling for the collisionality $\propto \mathrm{v}_{e}^{-4}$. Hence, heat-carrying electrons in the SOL are usually very weekly collisional, and the use Braginskii's equation for the parallel electron heat flux is totally unjustified. Over-population of the 'cold' boundary by supra-thermal electrons may require introduction of 'flux enhancement factors' for the parallel electron heat flux near the divertor target modelled in fluid codes, in addition to frequently used 'flux limits' for the upstream plasma, as was concluded in the kinetic simulations in [26].

Very low $E_{\mathrm{r}}$ values obtained in the code simulations presented in this paper may raise questions of whether all the controlling physics influencing the distribution of electric potential in the SOL has been properly incorporated into the code(s). Effects of very long connection lengths in the SOL rings next to the separatrix $(45 \mathrm{~m}$ from outer midplane to the target for AUG, on the first SOL ring), the influence of the private region on the adjacent SOL rings, influence of neutrals on the $E_{r}$ distributions, non-ambipolarity of perpendicular/radial plasma transport due to drifts, poloidal asymmetries of plasma potential distributions, will be subject to detailed analysis in the future. Cases will also be run on a much finer mesh (although first tests made on a SOLPS mesh with a doubled number of rings in the SOL did not result in any significant alterations of the solutions).

A separate issue, independent from the fluid modelling of the plasma edge, is the large $\mathrm{E}_{\mathrm{r}}$ compared to $\mathrm{T}_{\mathrm{e}}$ gradients, measured in the SOL by the probes. In AUG, JT-60U and TCV, $-\mathrm{eE}_{\mathrm{r}} / \nabla \mathrm{T}_{\mathrm{e}}$ ratios were $\sim 3$ (somewhat smaller in $\mathrm{JT}-60 \mathrm{U}, \approx 2.4$, but exceeding this value in $\mathrm{TCV}$ ). Since the primary physical parameter measured by the probes is the floating potential, the value of $-\mathrm{eE}_{\mathrm{r}} / \nabla \mathrm{T}_{\mathrm{e}}$ ratio obtained from the probe data depends critically on the value of the sheath potential drop Eq. (3). Since $e\left(V_{p}-V_{f}\right) / T_{e}=3$ was assumed, for flat SOL $V_{f}$ profiles 
as those in Figs. 3 (for ' $r$ '-values) and 7, one naturally obtaines $-\mathrm{eE}_{\mathrm{r}} / \nabla \mathrm{T}_{\mathrm{e}} \sim 3$. Equation 3, at the same time, is rather sensitive to the secondary electron emission coefficient $\delta$ which can increase dramatically under conditions of high electron temperature and high power fluxes onto the probe surface, up to the value $\delta \sim 1$ when the probe can go into self-emission. From a very limited database of measured $\mathrm{V}_{\mathrm{f}}$ and $\mathrm{T}_{\mathrm{e}}$ profiles considered in this paper no correlation between the magnitude of $\mathrm{T}_{\mathrm{e}}$ and $-\mathrm{eE}_{\mathrm{r}} / \nabla \mathrm{T}_{\mathrm{e}}$ ratios can however be established. This seems to suggest that the secondary electron emission didn't play a significant role in the measurements. However, it is known that secondary electron emission is very sensitive to surface conditions and topography, and reliable values of $\delta$ can be obtained only by a study of the material in the condition found in the particular experiment ([7], p.116). For a pure graphite surface (graphite was the material of the probe pins in all experiments described in this paper) and $\mathrm{T}_{\mathrm{e}}=50 \mathrm{eV}, \delta=0.544$ follows from Eq. (3.5) and Table 3.1 of [7] (pp.115116). This equation approximates very well the experimentally found normalized secondary electron emission coefficient given in Fig. 3.5 of this the same ref. Substituting this $\delta$ into Eq. (3) would give $e\left(V_{p}-V_{f}\right) / T_{e}=2.55$. For $T_{e}=100 \mathrm{eV}, \delta=0.777$ is obtained, giving $e\left(V_{p}-\right.$ $\left.\mathrm{V}_{\mathrm{f}}\right) / \mathrm{T}_{\mathrm{e}}=1.84$. This would be more in line with estimates made below in this section for high recycling divertor regimes. It has to be noted however, that a low energy electron released from a surface, when the magnetic field is rather oblique to the surface (which is typically the case in divertor configurations), has a finite Larmor radius and thus has a good chance of immediately returning to the surface within one Larmor period. The effective electron emission coefficient for fusion devices is thus sometimes taken to be zero, regardless of the original release rate ([7], p.114). Summarising, one has to conclude that the available data is insufficient to neither claim that the probe-invoked $-\mathrm{eE}_{\mathrm{r}} / \nabla \mathrm{T}_{\mathrm{e}}$ ratios were grossly overestimated by the neglect of higher secondary electron emission at positions closer to the separatrix, nor to expect that the effect of the secondary electron emission can be neglected. Its influence on the experimental results for the $-\mathrm{eE}_{\mathrm{r}} / \nabla \mathrm{T}_{\mathrm{e}}$ ratio remains an open question.

In contrast to the Langmuir probe data, $E_{\mathrm{r}}$ invoked from the Doppler reflectometry measurements on AUG, discussed in Section 2, combined with the $T_{\mathrm{e}}$ profile measured by the YAG laser would imply significantly smaller $-\mathrm{eE}_{\mathrm{r}} / \nabla \mathrm{T}_{\mathrm{e}}$ ratios, $<1$. The cause of the difference between the two methods of evaluating these ratios is presently not understood. It has to be pointed out, however, that neither Langmuir probe nor Doppler reflectometer technique allows one to measure $E_{r}$ directly. Evaluation of this quantity from the reflectometer data depends on the knowledge of the phase velocity of plasma density fluctuations. Its evaluation from Langmuir probe measurements depends on the theoretical relation between floating potential $\mathrm{V}_{\mathrm{f}}$ measured by the probe and plasma potential $\mathrm{V}_{\mathrm{p}}$, as discussed above, and on the magnitude of measured $T_{e}$. Increased secondary electron emission in the 'near SOL' can reduce the discrepancy between the probe and reflectometer $E_{\mathrm{r}}$ values, but is unlikely to eliminate it completely.

Large $-\mathrm{eE}_{\mathrm{r}} / \nabla \mathrm{T}_{\mathrm{e}}$ ratios, of order 3 , with $\mathrm{T}_{\mathrm{e}}$ being the upstream electron temperature, measured near the outer midplane position, rather than target temperature, are inconsistent with simple estimates that can be obtained for high-recycling SOLs. In the SOL, radial electric field is determined mainly by parallel plasma transport and interaction with the target. The upstream plasma potential is related to the target potential via the Debye sheath and magnetic presheath (MPS) drops at the target and the potential difference spread along the field line:

$V_{p, \text { upstream }}=V_{p, \text { target }}+\Delta V_{\text {Debye }}+\int E_{\|} d s_{\|}$, 
where $\Delta \mathrm{V}_{\text {Debye }}$ is the potential drop near the target due to the Debye sheath and MPS, $\mathrm{E}_{\|}$is the parallel electric field, and the integral is taken from the plasma position near the target, but outside of the MPS which is of width a few ion Larmor radii ([7], p.99), to the upstream position, e.g., near the outer midplane. The parallel electric field in turn can be expressed as:

$$
E_{\|}=-0.71 \nabla_{\|} T_{e} / e-\nabla_{\|} p_{e} / e n_{e}+j_{\|} / \sigma_{\|},
$$

following from the parallel electron force balance. The first term on the right hand side (RHS) of this equation describes the electron thermo-force; coefficient 0.71 is correct for singly charged ions, but is lower for higher charge states.

For the simplest SOL model with no neutral ionization, poloidally constant $T_{e}$ and plasma potential $\mathrm{V}_{\mathrm{p}}$, no parallel currents etc., one expects small $\mathrm{E}_{\|}$and $\Delta \mathrm{V}_{\text {Debye }} \sim 3 \mathrm{~T}_{\mathrm{e}} / \mathrm{e}$, yielding $-\mathrm{eE}_{\mathrm{r}} / \nabla \mathrm{T}_{\mathrm{e}} \sim 3$, which would be in agreement with some of the experiments showing similar $-\mathrm{eE}_{\mathrm{r}} / \nabla \mathrm{T}_{\mathrm{e}}$ ratios. In most of the experiments, however, strong recycling in the divertor results in a much lower target than upstream $\mathrm{T}_{\mathrm{e}}$. Negative parallel current density (driven by electrons) directed towards the outer target from the inner, provided the outer target is hotter (which is the most typical case), reduces upstream potential due to the contribution from the $\mathrm{j}_{\|} / \boldsymbol{\sigma}_{\|}$term. It however also reduces the target sheath drop $\Delta \mathrm{V}_{\text {Debye }}$, and these two effects partly cancel each other out. In the absence of plasma-neutral interactions and cross-field drifts (an idealistic case), the plasma pressure drops by $\sim$ factor 2 towards the target, an effect compensated by the plasma acceleration towards the target up to the ion sound speed (thus, contributing to the 'kinetic pressure'). This raises the plasma potential upstream with respect to the target potential due to the contribution of the $-\nabla p_{e} / \mathrm{en}_{\mathrm{e}}$ term. Interaction with neutrals can lead to more substantial pressure drop, resulting in the detachment of the plasma from the target (see e.g. [27]). The influence of neutral ionization and charge-exchange processes on the upstream $E_{r}$ profile via radial variation of the $-\nabla p_{e} / e_{e}$ term contributions (parallel gradient implied) is presently being assessed in dedicated EDGE2D code runs. The estimate given below which takes into account only the sheath drop $\Delta \mathrm{V}_{\text {Debye }} \sim 3 \mathrm{~T}_{\mathrm{e}} / \mathrm{e}$ and electron thermo-force and ignores the $-\nabla \mathrm{p}_{\mathrm{e}} / \mathrm{en}_{\mathrm{e}}$ term, gives a rough estimate for the radial electric field provided there is no strong non-ambipolarity of the radial plasma transport:

$e E_{r} \sim-3 \nabla T_{e, \text { target }}-0.71 \nabla\left(T_{e}-T_{e, \text { target }}\right)$,

where the gradient is taken upstream of the target along the radial direction, and target $T_{e}$ values are projected along field lines upstream. For the case of $\mathrm{T}_{\mathrm{e}, \text { target }}$ equal to the upstream electron temperature, this formula predicts $-\mathrm{eE}_{\mathrm{r}} / \nabla \mathrm{T}_{\mathrm{e}} \sim 3$, as pointed out above. For realistic cases with lower target temperatures, e.g. being only $\sim 1 / 2$ of the upstream ones, one obtains: $-\mathrm{eE}_{\mathrm{r}} / \nabla \mathrm{T}_{\mathrm{e}} \sim 1.85$. Ratios $-\mathrm{eE}_{\mathrm{r}} / \nabla \mathrm{T}_{\mathrm{e}} \sim 1.5$ following from some experiments (JET, Alcator C-Mod) may therefore be considered as not being in a strong disagreement with simple estimates. At the same time, $-\mathrm{eE}_{\mathrm{r}} / \nabla \mathrm{T}_{\mathrm{e}}$ ratios $\sim 3$ cannot be explained unless one assumes strong non-ambipolarity of the radial plasma transport or that the potential drop near the target is $>3 \mathrm{~T}_{\mathrm{e} \text {,target }} / \mathrm{e}$. The latter would be in line with the leading assumption about the cause of the discrepancies between simulated and experimental $T_{e}$ at the target and $E_{r}$ in the SOL, relating both to the presence of a significant fraction of supra-thermal electrons at the target. It is probable that these, energetic electrons can contribute not only to the target heat flux but also to the Debye sheath formation. This would require electrons from upstream with velocities in the $3-5 v_{T_{e}}$ range, responsible for the bulk of the parallel heat flux, to cascade 
down the energy scale via collisions with slower electrons on their way to the target, and to contribute significantly to the energy spectrum around $3 \mathrm{~T}_{\mathrm{e}, \text { target }}$, responsible for the formation of the sheath. However, the notion of 'electron temperature' with respect to the near target electron component may then be ill defined, as the electron energy distribution may be far from Maxwellian.

\section{Summary}

The $E_{r}$ profiles in the SOL simulated with the two widely used edge codes - SOLPS and EDGE2D, are in a disagreement with experimental data obtained in ASDEX Upgrade (AUG) and JET. They also contradict the supporting experimental evidence obtained with reciprocating Langmuir probes on JT-60U and TCV. Experimental profiles exhibit a positive $E_{r}$ throughout the SOL, with the plasma electric potential $V_{p}$ falling together with electron temperature $\mathrm{T}_{\mathrm{e}}$, and with the ratio $-\mathrm{eE}_{\mathrm{r}} / \nabla \mathrm{T}_{\mathrm{e}}$ varying between 1.6 and 5 . In contrast, in the modelling with Monte-Carlo neutrals, $V_{p}$ profiles are found to be fairly flat in most of the $\mathrm{SOL}$. For the same magnetic configuration and input power levels, the simulated $\mathrm{E}_{\mathrm{r}}$ rises with the decrease in the separatrix density. Nevertheless, reducing density in the simulations didn't produce large enough $\mathrm{E}_{\mathrm{r}}$ values relative to $-\nabla \mathrm{T}_{\mathrm{e}} / \mathrm{e}$ that would be consistent with the experiment. Further modelling will be performed with both fluid and Monte-Carlo neutrals in order to understand the origin of a difference between these cases and to check whether all the controlling physics influencing the $\mathrm{V}_{\mathrm{p}}$ distribution in the SOL has been included and/or correctly implemented in the codes.

In contrast to large $-\mathrm{eE}_{\mathrm{r}} / \nabla \mathrm{T}_{\mathrm{e}}$ ratios following from Langmuir probe measurements, Doppler reflectometer measurements in the standard Ohmic regime of AUG seem to indicate low values of this ratio in the SOL, assuming the phase velocity of density fluctuations measured by the reflectometer is approximately zero. There is, on the other hand, no direct conflict between $E_{r}$ values coming from the reflectometer and Langmuir probe data. Measured $E_{r}$ values are similar, but the probe position was further away from the separatrix.

Discrepancies between probe measured and code simulated $-\mathrm{eE}_{\mathrm{r}} / \nabla \mathrm{T}_{\mathrm{e}}$ ratios are consistent with the recently made conclusion, based on the benchmarking of the SOLPS code against the AUG experiment, that fluid codes tend to underestimate $T_{\mathrm{e}}$ in the divertor. This is likely to be explained by the presence of supra-thermal electrons originating from the upstream (e.g. midplane), which are usually only weakly collisional and cannot be described by fluid codes. The final answer will only become available after incorporation of kinetic effects into the present-day fluid codes enabling one to switch these effects on and off in the code runs simulating well-diagnosed experimental plasmas.

It is at present not clear whether the extra parallel electron power flux to the divertor carried by energetic electrons and the consequent rise in the target $T_{e}$, can be solely responsible for the discrepancies in $-\mathrm{eE}_{\mathrm{r}} / \nabla \mathrm{T}_{\mathrm{e}}$ ratios, via the mechanism of Debye sheath and magnetic presheath formation, with the potential drop being $\sim 3 \mathrm{~T}_{\mathrm{e}} / \mathrm{e}$ at the target. It is also possible that a large fraction of supra-thermal electrons from the upstream cascade down the energy scale on their approach to the target, increasing the potential drops at the target, thereby providing an extra increase of $E_{r}$ in the SOL. Under such conditions the correct evaluation of $T_{e}$ from Langmuir probes may become problematic due to strong deviations of the electron distribution function from Maxwellian. Experimental evidence from AUG, JT-60U and TCV, where $-\mathrm{eE}_{\mathrm{r}} / \nabla \mathrm{T}_{\mathrm{e}}$ ratios of order 2.5 and above (up to 5) were observed, indicate the possibility 
of energetic electrons directly influencing the target Debye sheath. It is also possible, however, that the probe-invoked $\mathrm{E}_{\mathrm{r}}$ values can be overestimated due to the assumption $\mathrm{e}\left(\mathrm{V}_{\mathrm{p}}-\right.$ $\left.V_{f}\right) / T_{e}=3$ used for deriving radial electric field from $T_{e}$ and $V_{f}$ measurements. The difference $\left(\mathrm{V}_{\mathrm{p}}-\mathrm{V}_{\mathrm{f}}\right)$ is sensitive to the adopted secondary electron emission coefficient $\delta$. The ratio $\mathrm{e}\left(\mathrm{V}_{\mathrm{p}}\right.$ $\left.-\mathrm{V}_{\mathrm{f}}\right) / \mathrm{T}_{\mathrm{e}}=3$ corresponds to the adopted value of $\delta=0.3$. In reality, $\delta$ can be lower or higher than 0.3 depending on the local electron temperature and the probe surface conditions. Under all circumstances, however, $\delta$ is expected to rise with the increase in $\mathrm{T}_{\mathrm{e}}$. Due to the uncertainty in the values of $\delta$, it is at present not possible to estimate the extent of a possible downward correction for the $-\mathrm{eE}_{\mathrm{r}} / \nabla \mathrm{T}_{\mathrm{e}}$ ratios caused by secondary electron emission.

A large underestimate of $\mathrm{E}_{\mathrm{r}}$ in the SOL by the present-day edge fluid codes is related with another known problem: the codes consistently underestimate parallel ion flows in the SOL measured with double-sided Langmuir probes ('Mach probes'). In the experiment, the radial electric field, together with the ion pressure gradient, is found to be one of the drivers for the part of the parallel ion flow which is dependent on the direction of the toroidal magnetic field, under the assumption that the ion Pfirsch-Schlüter flow is the major contributor to the measured ion flow $[21,22,28]$. An underestimate of $E_{r}$ in the codes therefore directly contributes to the discrepancy between simulated and experimental parallel ion flows. According to estimates, the inclusion of correct, experimental $\mathrm{E}_{\mathrm{r}}$ values in the theoretical formulas for the parallel ion Mach number can completely eliminate the difference between field-dependent parts of simulated and experimental flows in JET and significantly reduce the flows discrepancy in the AUG case [9].

\section{References}

[1] Chankin A V, Coster D P, Dux R, et al., Plasma Phys. Control. Fusion 48 (2006) 1839.

[2] Chankin A V, Coster D P, Dux R, et al., 'Comparison between measured divertor parameters in ASDEX Upgrade and SOLPS code solutions', paper P1-40 presented at the $17^{\text {th }}$ PSI Conference, Hefei, May 22-26 (2006), accepted for publication in J. Nucl. Mater.

[3] Chankin A V, Coster D P, Dux R, et al, 'Critical issues identified by the comparison between experimental and SOLPS modelling on ASDEX Upgrade', in Proc. of the $21^{\text {st }}$ IAEA Conference, Fusion Energy, Chengdu, China, October 16-21 2006, (CD-ROM), paper IAEA-CN-149/TH/P6-15, Vienna, 2006, IAEA.

[4] Braginskii S I, Review of Plasma Physics (Consultants Bureau, New York, 1965), Vol. 1, p. 205.

[5] Batishchev O V, Krasheninnikov S I, Catto Peter J, et al., Phys. Plasmas 4 (1997) 1672.

[6] Chodura R, Contrib. Plasma Phys. 28 (1998) 4/5, 303.

[7] Stangeby P C, in The Boundary of Magnetic Fusion Devices, IOP Publishing, Bristol (2000).

[8] Coster D P, Bonnin X, Chankin A, et al., 'Integrated modelling of material migration and target plate power handling at JET', in Proc. of the $20^{\text {th }}$ IAEA Conference, Fusion Energy, Vilamoura, Portugal, November 1-6 2004, Chengdu, China, October 16-21 2006, (CDROM), paper IAEA-CN-116/TH/P5-18, Vienna, 2006, IAEA.

[9] Chankin A V, Coster D P, Asakura N., et al., 'The Role of Radial Electric Field in Driving Parallel Ion Flow in the Scrape-off Layer of Divertor Tokamaks', submitted for publication in Nuclear Fusion.

[10] Coster D P, Chankin A V, Conway G D, et al., proc. of $32^{\text {nd }}$ EPS Conference on Plasma Phys. Tarragona, 27 June - 1 July 2005 ECA Vol.29C, P-1.008 (2005). 
[11] Kukushkin A S, Pacher H D, Pacher G W, Janeschitz G, Coster D, Loarte A and Reiter D, Nucl. Fusion 43 (2003) 716.

[12] Erents S K, Pitts R A, Fundamenski W, Gunn J P and Matthews G F, Plasma Phys. Control. Fusion 46 (2004) 1757.

[13] Erents S K, Fundamenski W, Corrigan G, Matthews G F, Zagorski R, 'EDGE2D modelling of JET and ITER Including the Combined Effect of Guiding Centre Drifts and an Edge Transport Barrier', paper P3-6 presented at the $17^{\text {th }}$ PSI Conference, Hefei, May 22-26 (2006), accepted for publication in J. Nucl. Mater.

[14] Müller H.W., Bobkov V., Rohde V., et al., proc. of $32^{\text {nd }}$ EPS Conference on Plasma Phys. Tarragona, 27 June - 1 July 2005 ECA Vol.29C, P-1.009 (2005).

[15] Müller H.W., Bobkov V., Herrmann A., et al., 'Deuterium plasma flow in the scrape-off layer of ASDEX Upgrade', paper P3-15 presented at the $17^{\text {th }}$ PSI Conference, Hefei, May 22-26 (2006), accepted for publication in J. Nucl. Mater.

[16] Conway G, Schirmer J, Klenge S, Suttrop W, Holzhauer E and the ASDEX Upgrade Team, Plasma Phys. Control Fusion 46 (2004) 951.

[17] Scott B D, private communication (2006).

[18] Conway G, Schirmer J, Angioni C, et al., 'Study of Turbulence and Radial Electric Field Transitions in ASDEX Upgrade using Doppler Reflectometry', in Proc. of the $21^{\text {st }}$ IAEA Conference, Fusion Energy, Chengdu, China, October 16-21 2006, (CD-ROM), paper IAEA-CN-149/EX/2-1, Vienna, 2006, IAEA.

[19] Dong J Q, Horton W, and Kim J Y, Phys. Fluids B4 (1992) 1867.

[20] Stangeby P C, Phys. Fluids 27 (1984) 682.

[21] Asakura N, Sakurai S, Shimada M, Koide Y, Hosogane N, and Itami K, Phys. Rev. Lett. 84 (2000) 3093.

[22] Pitts R A, Horacek J, Wischmeier M, 'Parallel SOL flow in TCV', paper O-16 presented at the $17^{\text {th }}$ PSI Conference, Hefei, May 22-26 (2006), accepted for publication in J. Nucl. Mater.

[23] LaBombard B, Rice J E, Hubbard A E, et al., Nucl. Fusion 33 (2004) 1047.

[24] Rozhansky V, Kaveeva E, Voskoboynikov S, et al., Plasma Phys. Control. Fusion 48 (2006) 1425.

[25] Horacek J, Pitts R A, Stangeby P C, Batishchev O, Loarte A, J. Nucl. Mater 313-316 (2003) 931.

[26] Kukushkin A S, Runov A M, Contrib. Plasma Phys. 34 (1994) 2/3, 204.

[27] Loarte A, Monk R D, Martín-Solís J R, et al., Nucl. Fusion 38 (1998) 331.

[28] Asakura N, and ITPA SOL and divertor topical group, 'Understanding the SOL flow in L-mode Plasma on divertor tokamaks, and its influence on the plasma transport', paper R-5 presented at the $17^{\text {th }}$ PSI Conference, Hefei, May 22-26 (2006), accepted for publication in J. Nucl. Mater. 